\title{
QUASI-ISOMORPHISM INVARIANTS FOR TWO CLASSES OF FINITE RANK BUTLER GROUPS
}

\author{
D. ARNOLD AND C. VINSONHALER
}

(Communicated by Ron Solomon)

\begin{abstract}
A complete set of numerical quasi-isomorphism invariants is given for a class of torsion-free abelian groups containing all groups of the form $\mathscr{G}[\mathscr{A}]$, where $\mathscr{A}=\left(A_{1}, \ldots, A_{n}\right)$ is an $n$-tuple of subgroups of the additive rationals and $\mathscr{G}[\mathscr{A}]$ is the cokernel of the diagonal embedding $\cap A_{i} \rightarrow \bigoplus A_{i}$. This classification and its dual include, as special cases, earlier classifications of strongly indecomposable groups of the form $\mathscr{G}[\mathscr{A}]$ and their duals.
\end{abstract}

The purpose of this note is to show that the complete sets of quasi-isomorphism invariants for strongly indecomposable torsion-free abelian groups of the form $\mathscr{G}(\mathscr{A})$ or $\mathscr{G}[\mathscr{A}]$ given in [AV3, AV4] actually classify these groups without the strong indecomposability assumption and, in fact, classify a strictly larger class of groups. Let $\mathscr{A}=\left(A_{1}, \ldots, A_{n}\right)$ be an $n$-tuple of subgroups of the additive rationals $Q$. Then $\mathscr{G}(\mathscr{A})$ is the kernel of the summation map $A_{1} \oplus \cdots \oplus A_{n} \rightarrow \sum A_{i} \subseteq Q$ and $\mathscr{G}[\mathscr{A}]$ is the cokernel of the diagonal embedding $\bigcap A_{i} \rightarrow A_{1} \oplus \cdots \oplus A_{n}$. Groups of the form $\mathscr{G}(\mathscr{A})$ are dual to groups of the form $\mathscr{G}[\mathscr{A}]$ via a quasi-homomorphism duality for Butler groups, which is detailed in [AV4]. Thus it suffices to work with just one of these classes. We choose to focus on the $\mathscr{G}[\mathscr{A}]$ 's for the relatively minor reason that this class is usually studied in terms of pure subgroups, while the $\mathscr{G}(\mathscr{A})$ 's are studied in terms of homomorphic images; the latter seem to be marginally more difficult to handle.

The known invariants for these groups are the ranks of a relatively small collection of subgroups, which we describe following some additional definitions. Our treatment utilizes basic tools developed in [AV1-7] and summarized and refined in [HM]. Fuchs and Metelli [FM] have obtained similar results using different techniques. The paper [AV8] provides a survey of existing literature on the subject.

In contrast to the usual definition, we define a type as an isomorphism class of subgroups of $Q$. A subgroup $X$ of $Q$ can then be identified with the type $\{\alpha X \mid 0 \neq \alpha \in Q\}$ to which it belongs, and we will do this whenever the context leaves no room for confusion. If $g$ is an element of a torsion-free group $G$, then $\operatorname{type}(g)=\{\alpha \in Q \mid \alpha g \in G\}$. If $X$ and $Y$ are subgroups of $Q$, we write

Received by the editors August 13, 1991.

1991 Mathematics Subject Classification. Primary 20K15.

The first author's research was supported in part by NSF grant DMS-9101000. The second author's research was supported in part by NSF grant DMS-9022730. 
$X \leq Y$ to indicate their relationship as types. Thus $X \subseteq Y$ implies $X \leq Y$, but not conversely. If $G$ is a torsion-free group and $X$ is a subgroup of $Q$ (or a type), denote $G(X)=\{g \in G \mid \operatorname{type}(g) \geq X\} ; G[X]=\bigcap\{\operatorname{ker} f \mid f: G \rightarrow X\}$, and for $\mathscr{M}$ a set of types, $G(\mathscr{M})=\sum\{G(X) \mid X \in \mathscr{M}\}$. In our notation, capital letters always denote groups, while script capitals denote $n$-tuples or sets of subgroups of $Q$. The only exception is the symbol $\mathscr{G}$, which is used to denote the formation of a group from an $n$-tuple, as in $\mathscr{G}[\mathscr{A}]$. Keeping in mind these conventions should help in avoiding the confusion inherent in the established notational use of [ ] and ( ) for our particular groups. For example, $\mathscr{G}(\mathscr{A})$ denotes a group formed from the $n$-tuple $\mathscr{A}$, while $G(\mathscr{M})$ denotes the subgroup of $G$ defined above.

Denote by $\Gamma$ the class of all Butler groups quasi-isomorphic to a direct sum of the form $G=\mathscr{G}\left[\mathscr{D}_{1}\right] \oplus \mathscr{G}\left[\mathscr{D}_{2}\right] \oplus \cdots \oplus \mathscr{G}\left[\mathscr{D}_{m}\right]$ for $\mathscr{D}_{1}, \ldots, \mathscr{D}_{m}$ tuples of subgroups of $Q$, such that:

(Г1) Each $\mathscr{G}\left[\mathscr{D}_{i}\right]$ is strongly indecomposable; and

(Г2) If rank $\mathscr{\mathscr { C }}\left[\mathscr{D}_{i}\right] \geq 2$, then $\sum\left\{G\left(A_{k}\right) \mid A_{k} \in \mathscr{D}_{i}\right\}$ is quasi-isomorphic to $\mathscr{G}\left[\mathscr{D}_{i}\right] \oplus C$, with $C$ completely decomposable.

Note that $\Gamma$ is closed under quasi-summands. We show in Proposition 6 that $\Gamma$ contains all groups of the form $\mathscr{G}[\mathscr{A}]$. The main theorem of the paper is the following.

Theorem 7. Let $G$ and $H$ be Butler groups in the class $\Gamma$. Then $G$ is quasiisomorphic to $H$ if and only if $\operatorname{rank} G(\mathscr{M})=\operatorname{rank} H(\mathscr{M})$ for each set of types $\mathscr{M}$ from the type lattice generated by typeset $G \cup$ typeset $H$.

This theorem, with the stronger hypothesis that $G$ and $H$ are strongly indecomposable groups of the form $\mathscr{G}[\mathscr{A}]$, appears in various forms in [AV4, FM, HM]. The rest of the paper is devoted to its proof.

An $n$-tuple $\mathscr{A}=\left(A_{1}, \ldots, A_{n}\right)$ of subgroups of $Q$ is called cotrimmed [Le] provided that for each $i, A_{i}=A_{i}+\bigcap_{j \neq i} A_{j}$. Equivalently, the canonical image of each $A_{i}$ in $\mathscr{G}[\mathscr{A}]$ is pure. It will be convenient to view $\mathscr{G}[\mathscr{A}]$ as a sum of these pure subgroups. Since multiplying the $n$-tuple $\mathscr{A}$ by a nonzero rational does not change the isomorphism class of $\mathscr{G}[\mathscr{A}]$ (see [R]), we can assume $1 \in A_{i}$ and denote by $a_{i}$ the image in $\mathscr{G}[\mathscr{A}]$ of the element $(0, \ldots, 1, \ldots, 0) \in A_{1} \oplus \cdots \oplus A_{i} \oplus \cdots \oplus A_{n}$ (that has a 1 in the $i$ th position and 0 's elsewhere). Then $\mathscr{G}[\mathscr{A}]=\sum A_{i} a_{i}$ and $a_{1}+\cdots+a_{n}=0$, while any proper subset of the $a_{i}$ 's is linearly independent. Our first two results are well known.

Lemma 1. Let $G$ be a Butler group and $X$ a type.

(a) $\operatorname{rank}(G(X)+G[X])-\operatorname{rank} G[X]$ is the rank of a maximal $X$-homogeneous completely decomposable quasi-summand of $G$.

(b) If $L$ is the lattice of types generated by typeset $G$, then $G[X]$ is the pure subgroup generated by $G(\mathscr{M})$, where $\mathscr{M}=\{Y \in L \mid Y \not X\}$.

(c) With $\mathscr{M}$ as in (b), $\operatorname{rank}(G(X)+G[X])-\operatorname{rank} G[X]=\operatorname{rank} G(\mathscr{M} \cup\{X\})-$ $\operatorname{rank} G(\mathscr{M})$.

Proof. For (a), see [AV1, Corollary 2.2]; Corollary 1.4 of [ARV] contains a version for representations of finite posets. Part (b) is due to Lady [La]. Part (c) follows from (b). 
Theorem 2 (see [AV3] or [AV6]). Let $\mathscr{A}=\left(A_{1}, \ldots, A_{n}\right)$ be a cotrimmed $n$ tuple of subgroups of $Q$ and $G=\mathscr{G}[\mathscr{A}]$. The following are equivalent.

(a) $G$ is strongly indecomposable.

(b) $\operatorname{rank} G\left(A_{i}\right)=1$ for $1 \leq i \leq n$.

(c) $\operatorname{End}(G)$ is isomorphic to a subring of $Q$.

An easy corollary of Theorem 2 will be used in the proof of the main theorem.

Corollary 3. Let $\mathscr{A}=\left(A_{1}, \ldots, A_{n}\right)$ be a cotrimmed $n$-tuple of subgroups of $Q$ such that $G=\mathscr{G}[\mathscr{A}]$ is strongly indecomposable. If $H$ is a torsion-free group such that $\operatorname{rank} H(\mathscr{M})=\operatorname{rank} G(\mathscr{M})$ for each subset $\mathscr{M}$ of $\left\{A_{1}, \ldots, A_{n}\right\}$, then $G$ is quasi-isomorphic to a subgroup of $H$.

Proof. Write $G=\sum A_{i} a_{i}$ with $a_{1}+\cdots+a_{n}=0$ in $G$. Theorem 2 and the hypothesis on ranks imply that there are elements $b_{i} \in H\left(A_{i}\right)$ such that $b_{1}+\cdots+b_{n}=0$, but any proper subset of the $b_{i}$ 's is independent. Then $a_{i} \rightarrow b_{i}$ defines a monic quasi-homomorphism of $G$ into $H$.

We complete the preliminaries with an additional known result. If $\mathscr{A}=$ $\left(A_{1}, \ldots, A_{n}\right)$ is an $n$-tuple of subgroups of $Q$ and $X$ is a subgroup of $Q$, we obtain an equivalence relation on the elements of $\mathscr{A}$ by calling $A_{i} X$ equivalent to $A_{j}$ provided $X \notin A_{i}+A_{j}$ and by extending via reflexivity and transitivity. Note that $\left\{A_{i}\right\}$ is always an $A_{i}$-equivalence class. The utility of this equivalence notion is indicated by the next theorem. To avoid cumbersome notation, we will frequently treat $n$-tuples as sets and vice versa. We also use $\dot{\simeq}$ to denote quasi-isomorphism.

Theorem 4 (see [AV2] or [AV6, Proof of Theorem 2.4]. Let $\mathscr{A}=\left(A_{1}, \ldots, A_{n}\right)$ be an $n$-tuple of subgroups of $Q$ and $G=\mathscr{G}[\mathscr{A}]$.

(a) If $X$ is any subgroup of $Q$, then $\operatorname{rank} G(X)+1$ is the number of $X$ equivalence classes in $\mathscr{A}$.

(b) If $\mathscr{A}$ is cotrimmed and $\mathscr{E}_{0}=\left\{A_{i}\right\}, \mathscr{E}_{1}, \ldots, \mathscr{E}_{r}$ are the $A_{i}$-equivalence classes in $\mathscr{A}$, then $G \dot{\sim} \mathscr{G}\left[\mathscr{E}_{1}^{\prime}\right] \oplus \cdots \oplus \mathscr{G}\left[\mathscr{E}_{r}^{\prime}\right]$, where, for each $1 \leq j \leq r$, $\mathscr{E}_{j}^{\prime}=\mathscr{E}_{j} \cup\left\{A_{i}\right\}$.

We are now ready to give an explicit description of the quasi-decomposition of $\mathscr{G}[\mathscr{A}]$ into strongly indecomposable summands.

Decomposition Algorithm for $G=\mathscr{G}[A]$. Assume $\mathscr{A}=\left(A_{1}, \ldots, A_{n}\right)$ is an $n$-tuple of subgroups of $Q$ and $G=\mathscr{G}[\mathscr{A}]$.

Let $\left\{A_{1}\right\}=\mathscr{E}_{0}, \mathscr{E}_{1}, \ldots, \mathscr{E}_{r}$ be the $A_{1}$-equivalence classes in $\mathscr{A}$. By Theorem $4(\mathrm{~b}), \mathscr{G}[\mathscr{A}]=\mathscr{G}\left[\mathscr{E}_{1}^{\prime}\right] \oplus \cdots \oplus \mathscr{\mathscr { S }}\left[\mathscr{E}_{r}^{\prime}\right]$ (up to quasi-isomorphism), where $\mathscr{E}_{i}^{\prime}=\mathscr{E}_{i} \cup\left\{A_{1}\right\}$. Assume $A_{2} \in \mathscr{E}_{i}^{\prime}$ for some (unique) $1 \leq i \leq r$. Write $\left\{A_{2}\right\}=\mathscr{D}_{0}, \mathscr{D}_{1}, \ldots, \mathscr{D}_{s}$ for the $A_{2}$-equivalence classes in $\mathscr{E}_{i}^{\prime}$, with $s \geq 1$. Again by Theorem $4(\mathrm{~b}), \mathscr{G}\left[\mathscr{E}_{i}^{\prime}\right] \dot{\sim} \mathscr{G}\left[\mathscr{D}_{1}^{\prime}\right] \oplus \cdots \oplus \mathscr{S}^{\prime}\left[\mathscr{D}_{s}^{\prime}\right]$, where $\mathscr{D}_{j}^{\prime}=\mathscr{D}_{j} \cup\left\{\bar{A}_{2}\right\}$. This in turn produces a further quasi-decomposition of $G$. Note that each of the summands $H_{i}=\mathscr{G}\left[\mathscr{D}_{i}^{\prime}\right]$ satisfies $\operatorname{rank} H_{i}\left(A_{2}\right)=1$ by Theorem $4(\mathrm{a})$. Iterate this procedure with $A_{3}, \ldots, A_{n}$. At the start of the $k$ th stage we have decomposed $G$ into quasi-summands of the form $\mathscr{G}[\mathscr{B}]$ where each $\mathscr{B}$ is a subtuple of $\mathscr{A}$ and the group $A_{k}$ belongs to precisely one of these tuples, say $\mathscr{B}_{0}$. We then further decompose $G$ by decomposing $\mathscr{G}\left[\mathscr{B}_{0}\right]$ via Theorem $4(\mathrm{~b})$, using $A_{k}$-equivalence classes. After $n$ iterations, we obtain a quasi-decomposition of $\mathscr{G}[\mathscr{A}]$ with some strong properties, as summarized in the next result. 
Proposition 5. Assume $\mathscr{A}=\left(A_{1}, \ldots, A_{n}\right)$ is a cotrimmed $n$-tuple of subgroups of $Q$ and $G=\mathscr{G}[\mathscr{A}]$. The decomposition algorithm above produces a quasidecomposition

$$
G \dot{\simeq} \mathscr{G}\left[\mathscr{D}_{1}\right] \oplus \cdots \oplus \mathscr{G}\left[\mathscr{D}_{m}\right]
$$

with the tuples $\mathscr{D}_{h}$ satisfying the following properties:

(a) Each $\mathscr{D}_{h}$ is a subtuple of $\mathscr{A}$ (not necessarily cotrimmed) with at least two components.

(b) If $h \neq i$, then $\mathscr{D}_{h} \cap \mathscr{D}_{i}$ is a subset of $\left\{A_{1}, \ldots, A_{n}\right\}$ containing at most one element.

(c) If $A_{k} \in \mathscr{D}_{h}$ and $G_{h}=\mathscr{G}\left[\mathscr{D}_{h}\right]$, then $\operatorname{rank} G_{h}\left(A_{k}\right)=1$.

(d) Each $\left.\mathscr{G}_{[} \mathscr{D}_{h}\right]$ is strongly indecomposable.

(e) For each $1 \leq h \leq m$ there is a strictly decending chain of tuples

$$
\mathscr{T}_{0}=\mathscr{A} \supset \mathscr{T}_{1} \supset \cdots \supset \mathscr{T}_{\mu(h)}=\mathscr{D}_{h} \text {, }
$$

with $\mathscr{T}_{j+1}=\left\{A_{k}\right\} \cup \mathscr{E}$ for some $A_{k} \in \mathscr{T}_{j}$ and some $A_{k}$-equivalence class $\mathscr{E} \neq\left\{A_{k}\right\}$ in $\mathscr{T}_{j}$.

Proof. Properties (a) and (b) follow readily from the construction of the algorithm. Property (c) is a consequence of Theorem 4: when the group $A_{k}$ is used to decompose the unique tuple to which it belongs at the start of the $k$ th stage, the resulting summands $H$ in which $A_{k}$ appears have $\operatorname{rank} H\left(A_{k}\right)=$ 1 , as noted in the description of the algorithm. Subsequent decompositions preserve this condition: each summand $H$ in which $A_{k}$ appears will have rank $H\left(A_{k}\right)$ equal to one. Property (d) is a consequence of (c) via Theorem 2. Since the tuples $\mathscr{D}_{i}$ are not necessarily cotrimmed, to apply Theorem 2 we need to observe that if $\left(B_{1}, \ldots, B_{k}\right)$ is the cotrimmed version of a $k$-tuple $\left(A_{1}, \ldots, A_{k}\right)\left(B_{i}=A_{i}+\bigcap_{j \neq i} A_{j}\right)$, then $A_{i} \subseteq B_{i}$. Thus, if $H=\mathscr{G}\left[A_{1}, \ldots, A_{k}\right]$ and $\operatorname{rank} H\left(A_{i}\right)=1$, then $\operatorname{rank} H\left(B_{i}\right)=1$ since $H=\mathscr{G}\left[B_{1}, \ldots, B_{k}\right]$ and $H\left(B_{i}\right) \subseteq H\left(A_{i}\right)$. Property (e) is a direct consequence of the design of the algorithm.

Proposition 6. Let $G=\mathscr{G}[\mathscr{A}]$ for some n-tuple $\mathscr{A}$ of subgroups of $Q$. Write $G \dot{\simeq} \mathscr{G}\left[\mathscr{D}_{1}\right] \oplus \cdots \oplus \mathscr{G}\left[\mathscr{D}_{m}\right]$ as in Proposition 5. If $G_{h}=\mathscr{G}\left[\mathscr{D}_{h}\right]$, then, for $k \neq h$, rank $\sum\left\{G_{h}\left(A_{i}\right) \mid A_{i} \in \mathscr{D}_{k}\right\}$ is either 0 or 1 . In particular, $G$ belongs to the class $\Gamma$.

Proof. Fix $k \neq h$ between 1 and $m$ and abbreviate $\bigcap \mathscr{D}_{h}=\bigcap\left\{A_{i} \mid A_{i} \in \mathscr{D}_{h}\right\}$. Using Proposition 5(e), the tuple $\mathscr{D}_{h}$ is obtained via a sequence of tuples $\mathscr{T}_{0}=$ $\mathscr{A}, \mathscr{T}_{1}, \ldots, \mathscr{T}_{\mu}=\mathscr{D}_{h}$, with $\mathscr{T}_{j}$ obtained as a subtuple of $\mathscr{T}_{j-1}$ by taking, for $A_{j(h)} \in \mathscr{T}_{j-1}$, an $A_{j(h)}$-equivalence class in $\mathscr{T}_{j-1}$ and adjoining $A_{j(h)}$. We will abbreviate $A_{j}=A_{j(h)}$. There is an analogous sequence $\left\{T_{j}^{\prime}\right\}$ for the tuple $\mathscr{D}_{k}$. Let $i(1) \geq 1$ be the smallest index $i$ such that $\mathscr{T}_{i} \neq \mathscr{T}_{i}{ }^{\prime}$. The subtuples $\mathscr{D}_{h}$ and $\mathscr{D}_{k}$ of $\mathscr{T}_{i(1)-1}$ belong to different $A_{i(1)}$-equivalence classes, since $\mathscr{T}_{i(1)} \neq$ $\mathscr{T}_{i(1)}^{\prime}$ are formed from different $A_{i(1)}$-equivalence classes as in Proposition 5(e). Thus, $\cap \mathscr{D}_{h}+\bigcap \mathscr{D}_{k} \geq A_{i(1)}$ by the definition of equivalence. Next let $i(2)$ be the smallest index $i>i(1)$ (if one exists) such that $\mathscr{T}_{i}$ does not contain $A_{i(1)}$. Then $A_{i(1)}$ and $\mathscr{D}_{h}$ are in distinct $A_{i(2)}$-equivalence classes in $\mathscr{T}_{i(2)-1}$ (where $\left.A_{i(2)} \in \mathscr{T}_{i(2)-1}\right)$, so that $A_{i(2)} \leq \bigcap \mathscr{D}_{h}+A_{i(1)}$. Continuing in this way, we obtain an increasing sequence of indices $i(1)<\cdots<i(t) \leq \mu$ such that 
$A_{i(j+1)} \leq A_{i(j)}+\bigcap \mathscr{D}_{h}$ and $A_{i(t)}$ belongs to $\mathscr{T}_{\mu}=\mathscr{D}_{h}$. Thus, we obtain the chain of inequalities

$$
\bigcap \mathscr{D}_{h}+\bigcap \mathscr{D}_{k} \geq A_{i(1)}+\bigcap \mathscr{D}_{h} \geq A_{i(2)}+\bigcap \mathscr{D}_{h} \geq \cdots \geq A_{i(t)} .
$$

Now suppose $A_{l}$ is an element of $\mathscr{D}_{k}$. Then

$$
\bigcap \mathscr{D}_{h}+A_{l} \geq \bigcap \mathscr{D}_{h}+\bigcap \mathscr{D}_{k} \geq A_{i(t)}
$$

If $\mathscr{E}_{1}$ and $\mathscr{E}_{2}$ are two $A_{l}$-equivalence classes in $\mathscr{D}_{h}$, then $\bigcap \mathscr{E}_{1}+\bigcap \mathscr{E}_{2} \geq A_{l}$ by the definition of equivalence. Since $\cap \mathscr{E}_{1}+\bigcap \mathscr{E}_{2} \geq \bigcap \mathscr{D}_{h}$, it follows that

$$
\bigcap \mathscr{E}_{1}+\bigcap \mathscr{E}_{2} \geq \bigcap \mathscr{D}_{h}+A_{l} \geq A_{i(t)}
$$

Thus, every $A_{l}$-equivalence class in $\mathscr{D}_{h}$ is a union of $A_{i(t)}$-equivalence classes. However, by Proposition 5(c) and Theorem 4, there are only two $A_{i(t)}$-equivalence classes in $\mathscr{D}_{h}$, namely, $\left\{A_{i(t)}\right\}$ and $\mathscr{D}_{h} \backslash\left\{A_{i(t)}\right\}$. Consequently, there are at most two $A_{l}$-equivalence classes in $\mathscr{D}_{h}$. Moreover, if there are exactly two $A_{l}$-equivalence classes, they must be $\left\{A_{i(t)}\right\}$ and $\mathscr{D}_{h} \backslash\left\{A_{i(t)}\right\}$. In this case, by 4(a), rank $G_{h}\left(A_{l}\right)=1$, where $G_{h}=\mathscr{G}\left[\mathscr{D}_{h}\right]$. Moreover, by the definition of $A_{l}$-equivalence, $G_{h}\left(A_{l}\right)$ is the pure subgroup generated by the image of $A_{i(t)}$ in $G_{h}$, namely, $G_{h}\left(A_{i(t)}\right)$. As we let $A_{l}$ range over the elements of $\mathscr{D}_{k}$, we see that $\sum\left\{G_{h}\left(A_{l}\right) \mid A_{l} \in \mathscr{D}_{k}\right\} \subseteq G_{h}\left(A_{i(t)}\right)$. Since the group $G_{h}\left(A_{i(t)}\right)$ has rank one by $5(\mathrm{c})$, the proof of the first assertion of the proposition is complete. To verify the assertion that $G$ belongs to $\Gamma$, note that condition $(\Gamma 1)$ holds for $G$ by Proposition $5(\mathrm{~d})$ and that condition $(\Gamma 2)$ is a direct consequence of the first part of Proposition 6.

By Proposition 6, $\Gamma$ contains all Butler groups quasi-isomorphic to groups of the form $\mathscr{G}[\mathscr{A}]$; however, there are many groups in $\Gamma$ which are not quasiisomorphic to a group of the form $\mathscr{G}[\mathscr{A}]$. For example, if $p_{1}, \ldots, p_{6}$ are distinct primes and $A_{i}$ is the smallest subring of $Q$ containing $p_{i}^{-1}$, then the group $G=\mathscr{G}\left[A_{1}, A_{2}, A_{3}\right] \oplus \mathscr{G}\left[A_{4}, A_{5}, A_{6}\right]$ is not quasi-isomorphic to a $\mathscr{G}[\mathscr{A}]$ (see [FM, Example 2.5]). But $G$ belongs to $\Gamma$ because, for instance, $\operatorname{Hom}\left(A_{i}, \mathscr{G}\left[A_{4}, A_{5}, A_{6}\right]\right)=0$ if $1 \leq i \leq 3$, so that $G\left(A_{1}\right)+G\left(A_{2}\right)+G\left(A_{3}\right)=$ $\mathscr{G}\left[A_{1}, A_{2}, A_{3}\right]$ and $(\Gamma 2)$ is satisfied.

We are ready for the main theorem.

Theorem 7. Let $G$ and $H$ be Butler groups belonging to the class $\Gamma$. Then $G$ is quasi-isomorphic to $H$ if and only if $\operatorname{rank} G(\mathscr{M})=\operatorname{rank} H(\mathscr{M})$ for each set of types $\mathscr{M}$ from the type lattice generated by typeset $G \cup$ typeset $H$.

Proof. The only if direction is clear. For the converse, by definition of $\Gamma$, we have $G \dot{\sim} \mathscr{G}\left[\mathscr{D}_{1}\right] \oplus \cdots \oplus \mathscr{G}\left[\mathscr{D}_{m}\right]$ with the $\mathscr{D}_{i}$ tuples of subgroups of $Q$ such that $(\Gamma 1)$ and $(\Gamma 2)$ are satisfied. It is easy to check that, without loss of generality, we may take the $\mathscr{D}_{i}$ 's to be cotrimmed. In this case, the lattice generated by typeset $G$ is the same as the lattice generated by the entries of the $\mathscr{D}_{i}$ 's ([Le] or [FM]). Abbreviate $G_{i}=\mathscr{G}\left[\mathscr{D}_{i}\right]$. By $(\Gamma 1)$ each $G_{i}$ is strongly indecomposable. Let $C$ be the direct sum of the $G_{i}$ 's which have rank one. By Lemma $1(\mathrm{c})$ and the hypotheses, for each type $X$ we have

$$
\operatorname{rank}(G(X)+G[X])-\operatorname{rank} G[X]=\operatorname{rank}(H(X)+H[X])-\operatorname{rank} H[X] .
$$


It follows from Lemma 1 (a) that $C$ is quasi-isomorphic to the direct sum of the rank one summands in a quasi-decomposition of $H$ into strongly indecomposable groups. As a consequence we may write $G \dot{\simeq} G^{\prime} \oplus C$ and $H \doteq H^{\prime} \oplus C$ where $G^{\prime}$ and $H^{\prime}$ are again in $\Gamma$ and have no rank one quasi-summands. In addition, since $\operatorname{rank} G(\mathscr{M})=\operatorname{rank} G^{\prime}(\mathscr{M})+\operatorname{rank} C(\mathscr{M})$ for each set of types $\mathscr{M}$, the groups $G^{\prime}$ and $H^{\prime}$ inherit the hypotheses of the theorem. Thus we may reduce to the case where $G$ and $H$ have no rank one quasi-summands. In particular, rank $\mathscr{G}\left[\mathscr{D}_{i}\right] \geq 2$ for each $i$, so that the quasi-isomorphism in $(\Gamma 2)$ holds for each $i$.

Write $\mathscr{D}_{1}=\left(A_{1}, \ldots, A_{k}\right)$ and denote $G^{\prime}=G\left(\mathscr{D}_{1}\right)=\sum_{i=1}^{k} G\left(A_{i}\right)$ and $H^{\prime}=$ $H\left(\mathscr{D}_{1}\right)=\sum_{i=1}^{k} H\left(A_{i}\right)$. By hypothesis, the ranks of $G^{\prime}$ and $H^{\prime}$ are equal. Moreover, by $(\Gamma 2), G^{\prime} \dot{\sim} G_{1} \oplus C$, where $C$ is a completely decomposable group. We will show that $H^{\prime} \dot{\sim} H_{1} \oplus C$ for some subgroup $H_{1}$ of $H^{\prime}$. Let $X$ be the type of a nonzero rank one summand of $C$. The group $G^{\prime}$ is a homomorphic image of $\bigoplus_{i=1}^{k} G\left(A_{i}\right)$, so for some $1 \leq i \leq k$ there is a nonzero map from $G\left(A_{i}\right)$ to $X$. For convenience take $i=1$. Then $X \geq A_{1}$, so that $G^{\prime}(X) \subseteq$ $G(X)=G\left(A_{1}\right) \cap G(X) \subseteq G^{\prime}(X)$ and $G(X)=G^{\prime}(X)$. Similarly, $H(X)=H^{\prime}(X)$. Clearly, $G^{\prime}[X]$ contains the pure subgroup $G^{\prime \prime}$ generated by $\sum_{i=1}^{k} G\left(A_{i}\right)[X]$. Moreover, $G^{\prime} / G^{\prime \prime}$ is a homomorphic image of $\bigoplus_{i=1}^{k} G\left(A_{i}\right) /\left(G\left(A_{i}\right)[X]\right)$ and, hence, has outer type less than or equal to $X$. It follows that $G^{\prime}[X]=G^{\prime \prime}$. Similarly, $H^{\prime}[X]$ is purely generated by $\sum_{i=1}^{k} H\left(A_{i}\right)[X]$. By Lemma $1(\mathrm{~b})$, each $G\left(A_{i}\right)[X]$ and $H\left(A_{i}\right)[X]$ is purely generated by $G\left(\mathscr{M}_{i}\right)$ for $\mathscr{M}_{i}$ a set of types $T$ satisfying $A_{i} \leq T \notin X$. If $\mathscr{M}=\bigcup \mathscr{M}_{i}$ then $G^{\prime}[X]$ is the pure subgroup generated by $G(\overline{\mathscr{M}})$ and $H^{\prime}[X]$ is the pure subgroup generated by $H(\mathscr{M})$. We have established the following equalities:

$$
\begin{gathered}
\operatorname{rank} G^{\prime}(X)=\operatorname{rank} G(X)=\operatorname{rank} H(X)=\operatorname{rank} H^{\prime}(X) ; \\
\operatorname{rank} G^{\prime}[X]=\operatorname{rank} G(\mathscr{M})=\operatorname{rank} H(\mathscr{M})=\operatorname{rank} H^{\prime}[X] ; \\
\operatorname{rank}\left(G^{\prime}(X)+G^{\prime}[X]\right)=\operatorname{rank} G(\mathscr{M} \cup\{X\})=\operatorname{rank} H(\mathscr{M} \cup\{X\}) \\
=\operatorname{rank}\left(H^{\prime}(X)+H^{\prime}[X]\right) .
\end{gathered}
$$

By Lemma $1($ a), $\operatorname{rank} G(\mathscr{M} \cup\{X\})-\operatorname{rank} G(\mathscr{M})=\operatorname{rank} H(\mathscr{M} \cup\{X\})-\operatorname{rank} H(\mathscr{M})$ is the rank of a maximal $X$-homogeneous completely decomposable quasisummand of $G^{\prime}$ and of $H^{\prime}$. It follows that $H^{\prime} \dot{\simeq} H_{1} \oplus C$, where $C$ is a completely decomposable group such that $G^{\prime} \dot{\simeq} G_{1} \oplus C$.

The next step is to show that $G_{1}$ embeds into $H_{1}$. Note that for any subset $\mathscr{M}$ of $\left\{A_{1}, \ldots, A_{k}\right\}$, rank $G_{1}(\mathscr{M})=\operatorname{rank} H_{1}(\mathscr{M})$, since $\operatorname{rank} G^{\prime}(\mathscr{M})=$ $\operatorname{rank} G(\mathscr{M})=\operatorname{rank} H(\mathscr{M})=\operatorname{rank} H^{\prime}(\mathscr{M})$ and $\operatorname{rank} G^{\prime}(\mathscr{M})=\operatorname{rank} G_{1}(\mathscr{M})+$ rank $C(\mathscr{M})$. Therefore, $G_{1}$ is quasi-isomorphic to a subgroup of $H_{1}$ by Corollary 3. Similarly, each $G_{i}=\mathscr{G}\left[\mathscr{D}_{i}\right]$ is quasi-isomorphic to a subgroup of $H$. By symmetry, any strongly indecomposable quasi-summand of $H \in \Gamma$ is quasi-isomorphic to a subgroup of $G$. It follows that there is a nonzero map $G_{i} \rightarrow H \rightarrow G$. The image of this map has nonzero projection onto some $G_{j}$. By the same reasoning, we can then obtain a nonzero composition

$$
G_{i} \rightarrow H \rightarrow G \rightarrow G_{j} \rightarrow H \rightarrow G \rightarrow G_{k}
$$

for some $k$. If we continue this process, eventually one of the subscripts on the $G$ 's will repeat. At this point, for some index $l$, we will have a nonzero 
composition $G_{l} \rightarrow H \rightarrow G_{l}$ that is a quasi-automorphism of $G_{l}$, since $G_{l}$ strongly indecomposable implies $\operatorname{End}\left(G_{l}\right) \subset Q$ by Theorem 2. As a consequence, we may write $G \dot{\simeq} G_{l} \oplus G^{\prime}$ and $H \dot{\check{ }} G_{l} \oplus H^{\prime}$ for some groups $G^{\prime}$ and $H^{\prime}$; however, the class $\Gamma$ is closed under quasi-summands, so that $G^{\prime}$ and $H^{\prime}$ belong to $\Gamma$. As noted previously, the quasi-direct decompositions $G \dot{\simeq} G_{l} \oplus G^{\prime}$ and $H \dot{\simeq} G_{l} \oplus H^{\prime}$, along with the hypothesis of the theorem, imply that $\operatorname{rank} G^{\prime}(\mathscr{M})=\operatorname{rank} H^{\prime}(\mathscr{M})$ for each set of types $\mathscr{M}$ from the lattice generated by typeset $G^{\prime} \cup$ typeset $H^{\prime} \subseteq$ typeset $G \cup$ typeset $H$. The confluence of these remarks allows us to apply an induction on rank to $G^{\prime}$ and $H^{\prime}$, and the proof is complete.

Let $\Gamma^{\prime}$ be the class of all Butler groups quasi-isomorphic to groups of the form $G=\mathscr{G}\left(\mathscr{D}_{1}\right) \oplus \cdots \oplus \mathscr{G}\left(\mathscr{D}_{m}\right)$, where each $\mathscr{D}_{i}$ is a tuple of subgroups of $Q$, such that each $\mathscr{G}\left(\mathscr{D}_{i}\right)$ is strongly indecomposable; and if rank $\mathscr{G}\left(\mathscr{D}_{i}\right) \geq 2$, then $G / \cap\left\{G\left[A_{k}\right] \mid A_{k} \in \mathscr{D}_{i}\right\} \dot{\simeq} \mathscr{G}\left(\mathscr{D}_{i}\right) \oplus C$, with $C$ completely decomposable. Applying the duality of [AV4] immediately provides the following.

Corollary 8. Let $G$ and $H$ be Butler groups in the class $\Gamma^{\prime}$. Then $G$ and $H$ are quasi-isomorphic if and only if $\operatorname{rank}\left(\bigcap_{X \in \mathscr{M}} G[X]\right)=\operatorname{rank}\left(\bigcap_{X \in \mathscr{M}} H[X]\right)$ for each subset $\mathscr{M}$ of the lattice of types generated by typeset $G \cup$ typeset $H$.

Remark. Analogs of Theorem 7 and Corollary 8 hold in the context of representations of finite posets, a fact we note but do not prove. The interested reader can make the minor changes needed to obtain the (more general) proofs by referring to [AV6].

\section{REFERENCES}

[ARV] D. M. Arnold, F. Richman, and C. Vinsonhaler, Representations of finite posets and valuated groups, J. Algebra (to appear).

[AV1] D. M. Arnold and C. I. Vinsonhaler, Representing graphs for a class of torsion-free abelian groups, Abelian Group Theory, Gordon and Breach, London, 1987, pp. 309-332.

[AV2] _ Quasi-isomorphism invariants for a class of torsion-free abelian groups, Houston J. Math. 15 (1989), 327-339.

[AV3] _ Invariants for a class of torsion-free abelian groups, Proc. Amer. Math. Soc. 105 (1989), 293-300.

[AV4] _ Duality and invariants for Butler groups, Pacific J. Math. 148 (1991), 1-9.

[AV5] _ Pure subgroups of finite rank completely decomposable groups. II, Lecture Notes in Math., vol. 1006, Springer-Verlag, New York, 1983, pp. 97-143.

[AV6] _ Invariants for classes of indecomposable representations of finite posets, J. Algebra 147 (1992), 245-264.

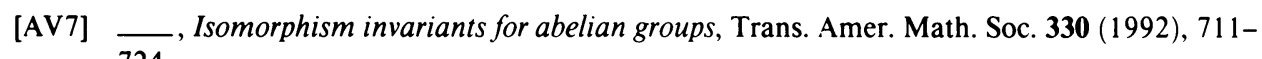
724.

[AV8] _ - Finite rank Butler groups, a survey of recent results, Proceedings of the Curacao Conference on Abelian groups (to appear).

[FM] L. Fuchs and C. Metelli, On a class of Butler groups, Manuscripta Math. 71 (1991), 1-28.

[HM] P. Hill and C. Megibben, The classification of certain Butler groups, J. Algebra (to appear).

[La] E. L. Lady, Extension of scalars for torsion free modules over Dedekind domains, Sympos. Math., vol. 23, Academic Press, New York, 1979, pp. 287-305. 
[Le] W. Y. Lee, Co-representing graphs for a class of torsion-free abelian groups, Ph.D. thesis, New Mexico State Univ., 1986.

[R] F. Richman, An extension of the theory of completely decomposable torsion-free abelian groups, Trans. Amer. Math. Soc. 279 (1983), 175-185.

Department of Mathematics, Baylor University, Waco, TeXas 76798-7328

E-mail address: ARNOLDD@BAYLOR.BITNET

Department of Mathematics, University of Connecticut, Storrs, Connecticut 062690001

E-mail address: VINSON@UCONNVM.BITNET 